\title{
Uso de Drogas e Bullying entre Adolescentes Brasileiros*
}

\author{
Bárbara de Oliveira Prado Sousa ${ }^{1}$ (D), Manoel Antônio dos Santos ${ }^{1, * *}$ (D), \\ Ana Carina Stelko-Pereira² (D), Erika de Cássia Lopes Chaves ${ }^{3}$ (D), \\ Denis da Silva Moreira ${ }^{3}$ (D), \& Sandra Cristina Pillon ${ }^{1}$ (D) \\ ${ }^{1}$ Universidade de São Paulo, São Paulo, SP, Brasil \\ ${ }^{2}$ Universidade Federal do Paraná, Curitiba, PR, Brasil \\ ${ }^{3}$ Universidade Federal de Alfenas, Alfenas, MG, Brasil
}

\begin{abstract}
RESUMO - Este estudo teve como objetivo avaliar possíveis relações entre uso de drogas e envolvimento em bullying entre adolescentes. Participaram 1.192 adolescentes escolares, que preencheram um formulário de informações sociodemográficas, DUSI R e Escala de Violência Escolar. Enquanto o consumo de álcool, cocaína e algum tipo de droga (exceto álcool e tabaco) foi associado a ser autor de bullying, o uso de algum tipo de droga (exceto álcool e tabaco) esteve associado a ser alvo (vítima); o caso de ser alvo/autor mostrou-se associado ao uso de maconha. Idade, localização da escola e religião foram fatores relevantes para a ocorrência de bullying. Ter 12 anos, ser ateu e morar na zona urbana aumentaram as chances de ser vítima, enquanto ter 15 anos e habitar a zona urbana elevaram as possibilidades de ser alvo/autor de bullying, assim como ter 13 anos, as de ser autor. Estar envolvido em situações de bullying em qualquer posição se mostrou associado ao uso de drogas.
\end{abstract}

PALAVRAS-CHAVE: drogas, bullying, adolescentes, saúde escolar, transtornos relacionados ao uso de substâncias

\section{Drug Use and Bullying among Brazilian Adolescents}

\begin{abstract}
This study aimed to evaluate possible relations between drug use and peer involvement in bullying among adolescents. A cross-sectional study was carried out with 1,192 school Brazilian adolescents. A sociodemographic information form, the DUSI R and the School Violence Scale were used. The consumption of alcohol, cocaine and some type of drug (except alcohol and tobacco) was associated with bullying author; the use of some type of drug (except alcohol and tobacco) was associated with bullying target (victim); and marijuana with bullying target/author. Being 12 years old, atheist and urban dwellers increased chances for victimization, while being 15 years old and living in the urban area, for being target/author; and 13 years, for being authors. Age, school location, and religion were relevant factors for the occurrence of bullying. Involvement in bullying situations in any position is associated with drug use.
\end{abstract}

KEYWORDS: drugs, bullying, adolescents, school health, substance-related disorders

O bullying é uma das formas mais comuns de violência em ambiente escolar e ocorre, geralmente, na fase da adolescência. Em termos conceituais, o bullying é definido como episódios de ações negativas e intencionais desencadeadas em um contexto relacional de desequilíbrio de poder, cuja expressão varia por serem de ordem psicológica, física ou sexual. São formas de intimidação a que adolescentes em idade escolar estão expostos, caracterizadas por provocações e ameaças que tornam o ambiente escolar hostil e sujeito a antagonismos. As formas de envolvimento nas situações de bullying compreendem: a condição de autor (agressor), alvo (vítima), alvo/autor (agressor e vítima simultaneamente) e testemunha (Olweus, 1993, 2013).

Já existe um corpus abundante de evidências derivadas de estudos que avaliaram isoladamente o fenômeno do bullying, com o intuito de minimizar seus efeitos deletérios na saúde dos adolescentes e reduzir a prevalência do fenômeno nas escolas. Esses estudos fornecem subsídios para a adoção

\footnotetext{
* Esse estudo é parte da tese de doutorado da primeira autora, intitulada "Comportamentos de risco, uso de substâncias psicoativas, bullying e problemas relacionados em adolescentes escolares” pela Universidade de São Paulo, a qual contou com o apoio da CAPES por meio de concessão de bolsa.

** E-mail: barbaraprado89@hotmail.com

- Submetido: 23/09/2016; Revisado: 22/03/2018; Aceito: 23/04/2018.
} 
de medidas preventivas de saúde pública que devem ser implementadas no contexto escolar (Forlim, Stelko-Pereira, \& Williams, 2014; Ttofi, Farrington, Lösel, \& Loeber, 2011) e familiar (Oliveira et al., 2018a, 2018b). Foram observadas associações positivas entre bullying e comportamentos de risco, tais como conflito com a lei (delinquência), porte ilegal de arma e uso de substâncias psicoativas (Bradshaw, Waasdorp, Goldweber, \& Johnson, 2013; Luukkonen, 2010; Pranjic \& Bajraktarevic, 2010; Schnohr \& Niclasen, 2006; Ttofi \& Farrington, 2008).

Adolescentes vítimas de bullying apresentam riscos potenciais de desenvolverem depressão, ansiedade, distúrbios do sono e prejuízos no ajustamento escolar. Já os adolescentes autores de bullying mostram risco elevado para uso de substâncias psicoativas, independentemente do tipo (Carlyle \& Steinman, 2007; Nansel, Craig, Overpeck, Saluja, \& Ruan, 2004; Tharp-Taylor, Haviland,\& D`Amico, 2009), bem como problemas acadêmicos e propensão a apresentar comportamentos violentos na vida adulta (Farrington \& Baldry, 2010).

Estudos longitudinais revelaram que comportamentos de bullying na infância são fatores de risco potencialmente associados ao uso de drogas no final da adolescência e início da idade adulta (Kim, Catalano, Haggerty, \& Abbott, 2011; Niemelä et al., 2011). Investigações internacionais que avaliaram a associação entre o envolvimento em bullying e o uso de drogas na adolescência constataram que ser autor ou vítima esteve relacionado ao uso de álcool, tabaco e outras drogas (Durand et al., 2013; Houbre, Tarquino, Thuillier, \& Hergott, 2006). Além disso, essa associação também foi observada em jovens que foram, simultaneamente, alvos e autores de bullying (Kaltiala-Heino, Rimpelä, Rantanen, \& Rimpelä, 2000; Radliff, Wheaton, Robisom, \& Morris, 2012).

Estudos realizados na Finlândia (Kaltiala-Heino et al., 2000) e nos Estados Unidos da América (EUA) (Radliff et al., 2012) revelaram que o indivíduo que foi alvo/autor de bullying tem três vezes mais chances de usar bebidas alcoólicas e sete vezes mais chances de usar drogas, assim como maior tendência ao uso de tabaco. Ter sido autor e vítima de bullying exclusivamente foi associado ao uso de tabaco, álcool, inalantes, maconha e, em alguns casos, de outras drogas (Durand et al., 2013; Houbre et al., 2006).

Um estudo que envolveu 78.333 estudantes nos EUA também apontou que adolescentes vítimas de bulllying apresentaram maiores chances de consumo de substâncias psicoativas, bem como padrões mais graves do uso de drogas (Radlliff et al., 2012). A coocorrência de comportamentos agressivos e uso de substâncias psicoativas também foi relacionada às tentativas fracassadas, por parte dos adolescentes, de enfrentamento das situações de vitimização e rejeição pelos pares (Carlyle \& Steinman, 2007).

No Brasil, nota-se escassez de estudos dedicados a avaliar a associação entre uso de drogas e a ocorrência do bullying, apesar dos fenômenos serem considerados relevantes para saúde pública (Horta et al., 2018; Zsila et al., 2017). Parte das pesquisas disponíveis consiste em estudos de revisão da literatura (Horta et al., 2018; Silva et al., 2016).
Dentro destes, os levantamentos epidemiológicos nacionais avaliaram, de modo isolado, apenas a prevalência do uso de substâncias psicoativas (Carlini et al., 2010; Malta et al., 2010) ou o envolvimento dos adolescentes em idade escolar em situações de bullying (Forlim et al., 2014). Desse modo, nota-se uma lacuna considerável no campo dos estudos empíricos nacionais que relacionam uso de drogas e bullying em adolescentes escolares.

Os resultados do último levantamento nacional sobre o consumo de drogas psicoativas entre estudantes do Ensino Fundamental e Ensino Médio apontaram altos índices de uso de drogas lícitas, sendo 60,5\% de álcool, 16,9\% de tabaco e $25,5 \%$ de qualquer droga (exceto álcool e tabaco) alguma vez na vida. Dentre os adolescentes que experimentaram ao menos uma vez na vida, $21,1 \%$ relataram ter usado álcool, $5,5 \%$ tabaco e $5,5 \%$ qualquer droga (exceto álcool e tabaco) no mês anterior à entrevista (Carlini et al., 2010).

Dados da Pesquisa Nacional de Saúde do Escolar PeNSE (Instituto Brasileiro de Geografia e Estatística, 2016) revelaram que $71,4 \%$ dos estudantes fizeram uso experimental de bebidas alcoólicas e $8,7 \%$ de outras drogas (Malta et al., 2010). Estudo realizado com 348 adolescentes do $6^{\circ}$ ao $9^{\circ}$ ano de duas escolas públicas de São Carlos, município do interior do estado de São Paulo, revelou que $16 \%$ haviam sido alvos, $23 \%$ alvo/autor e $11 \%$ autores de bullying (Forlim et al., 2014). Em ambas as investigações, os resultados apontaram índices preocupantes de bullying e de consumo de substâncias psicoativas, uma vez que são considerados problemas que podem resultar em comportamentos de alto risco, com diversas consequências negativas para a saúde dos adolescentes.

Os efeitos negativos resultantes do bullying na vida do adolescente se agravam ainda mais quando há o envolvimento com o uso de drogas (Luk, Wang, \& SimonsMorton, 2012). Essa sobreposição potencializa os riscos para o desenvolvimento de problemas psicossociais na adolescência (Valdebenito, Ttofi, \& Eisner, 2015). De modo geral, não existem critérios universais para estabelecer a relação de causalidade entre uso de drogas e bullying, porém pesquisadores sugerem algumas hipóteses: (1) o uso de drogas pode funcionar como mecanismo de enfrentamento frente a experiências estressantes vivenciadas durante a vida, incluindo o bullying no âmbito escolar; (2) vítimas de bullying podem se envolver no uso de drogas como uma forma de enfrentamento de experiências escolares negativas; (3) autores de bullying, por apresentarem fatores parentais estressores, percebem a escola como um ambiente hostil e envolto por uma atmosfera carregada, o que favorece o engajamento em hábitos e comportamentos que podem favorecer o uso de diversas substâncias psicoativas como um modo de enfrentamento das experiências adversas vivenciadas no ambiente escolar (Cook, Williams, Guerra, Kim, \& Sadek, 2010; Lazarus, 1993; Valdebenito et al., 2015).

No contexto brasileiro nota-se que pouca atenção tem sido dedicada à investigação da interface entre bullying e consumo de substâncias psicoativas. Revisão sistemática corroborou que a literatura ainda é incipiente em termos 
de estudos que mensuram o bullying no âmbito escolar no Brasil. Os resultados evidenciaram que as pesquisas realizadas estão concentradas nos estados de São Paulo e Rio Grande do Sul. Outro aspecto que se destaca entre os estudos brasileiros refere-se à falta de consenso sobre o conceito de violência escolar e à escassez de instrumentos padronizados que possibilitem a mensuração e melhor compreensão desse fenômeno no contexto nacional (Nesello et al., 2014).

Em busca realizada na Scientific Electronic Library Online (SciELO) em maio de 2016, com a palavra-chave bullying, foram encontrados 104 artigos de autores nacionais, dos quais apenas um avaliou a relação entre violência física, consumo de álcool e/ou de outras drogas e o envolvimento com o bullying em adolescentes escolares (Andrade et al., 2012). Nos demais estudos, essa relação foi mencionada somente nos achados da pesquisa, não tendo sido apresentada como parte do objetivo principal da investigação, uma vez que a proposta dessas investigações era apenas oferecer uma descrição do problema pesquisado, e não, dimensionar a magnitude de sua associação. Isso permite concluir que, apesar de mais de uma centena de artigos nacionais abordarem o uso de drogas e algumas facetas do bullying, a literatura é de fato incipiente na mensuração da relação desses fenômenos em amostras robustas de adolescentes escolares.

Dessa forma, pode-se afirmar que, apesar de o bullying ter sido por várias vezes analisado de forma isolada, até onde se pôde constatar nos estudos revisados, nenhuma investigação brasileira avaliou efetivamente a relação entre o consumo de drogas - uso de qualquer tipo de drogas, uso de drogas (exceto álcool e tabaco), álcool, tabaco, maconha e cocaína -e o envolvimento em bullying. Assim, o presente estudo teve por objetivo avaliar as possíveis relações entre uso de drogas e o envolvimento em bullying entre adolescentes escolares.

\section{MÉTODO}

Trata-se de um estudo de levantamento (survey) transversal, descritivo e de abordagem quantitativa. A pesquisa descritiva tem como objetivo mapear a distribuição de um fenômeno na população estudada. Em geral, busca determinar a frequência em que algo ocorre ou a relação entre duas variáveis, sendo tipicamente guiada por uma hipótese inicial. A principal hipótese que norteou o presente estudo foi: o bullying em adolescentes escolares está associado ao uso de substâncias psicoativas.

\section{Participantes}

Todas as escolas de um município de médio porte do sul de Minas Gerais foram convidadas a participar do estudo. De um total de 14 escolas, apenas uma instituição privada recusou-se a participar. Das escolas que compuseram o universo amostral, 10 eram públicas e três particulares; 11 estavam localizadas na zona urbana e duas na zona rural do município.

A população total de estudantes do município era de 4547 alunos matriculados no ano de 2013. A amostra necessária foi estimada em 1.572 alunos por meio de cálculo amostral (95\% de confiabilidade e $2 \%$ de precisão). Dessa amostra, $270(17,2 \%)$ alunos não responderam por completo o instrumento e $110(6,9 \%)$ devolveram em branco os questionários do estudo. Ao final, 1192 (75,8\%) estudantes participaram do estudo.

Os estudantes foram selecionados de acordo com os critérios de elegibilidade preestabelecidos, a saber: estar matriculado regularmente no $6^{\circ}$ ao $9^{\circ}$ ano do Ensino Fundamental e estar presente em sala de aula no momento da aplicação coletiva do questionário. Foram excluídos do estudo os adolescentes ausentes em sala de aula após três tentativas de coleta de dados, em dias diferentes, ou que estavam afastados da escola por qualquer motivo.

\section{Instrumentos}

Os instrumentos empregados foram: (1) informações sociodemográficas (sexo, idade, raça/etnia, religião, participação em festas/baladas, com quem reside, relacionamento familiar, série em que está matriculado, tipo de escola e localização da escola); (2) Drug Use Screening Inventory (DUSI R) (de Micheli \& Formigoni, 2000); (3) Escala de Violência Escolar - versão Estudantes (EVE) (Stelko-Pereira, Williams, \& Freitas, 2010).

O DUSI R é um questionário padronizado que avalia os problemas relacionados ao uso de álcool e outras drogas por adolescentes. Foi desenvolvido para identificar o uso de substâncias psicoativas no último mês, em resposta à necessidade prática e objetiva de um instrumento que avaliasse de forma eficiente os problemas associados ao uso de álcool e/ou outras drogas em adolescentes (Tarter, 1990). Estudos de validação do DUSI para o contexto nacional mostraram propriedades psicométricas satisfatórias, com sensibilidade de $80 \%$, especificidade de $90 \%$ e alfa de Cronbach 0,96 (de Micheli \& Formigoni, 2002).

Na presente pesquisa, avaliou-se os tipos de substâncias consumidas, tais como: álcool, tabaco e outras drogas (anfetaminas, ecstasy, cocaína, crack, maconha, alucinógenos, tranquilizantes, ansiolíticos, esteroides, inalantes e solventes).

O uso experimental de drogas foi avaliado com base no último mês antes da pesquisa. As variáveis relacionadas ao tipo de drogas foram categorizadas em seis grupos: (a) uso de qualquer substância (álcool, tabaco e outras drogas); (b) uso de drogas (exceto álcool e tabaco); (c) álcool; (d) tabaco; (e) maconha; (f) cocaína/crack.

A Escala de Violência Escolar - versão Estudantes (EVE-E) foi elaborada a partir do Questionário de Investigação de Prevalência de Violência Escolar (QIPVE). 
Esse instrumento foi desenvolvido com o objetivo de avaliar a violência física, psicológica/emocional e contra o patrimônio no ambiente escolar. $O$ instrumento é composto por 51 itens, com respostas do tipo Likert que variam da seguinte maneira: (0) nenhuma; (1) 1 ou 2 vezes; (2) 3 ou 4 vezes; (3) 5 ou 6 vezes; (4) 7 vezes ou mais. Um estudo de validação do EVE-E para o cenário brasileiro mostrou consistência interna com excelente nível de confiabilidade - alfa de Cronbach 0,95 (Stelko-Pereira et al., 2010).

Não há um critério padronizado que permite estabelecer uma pontuação mínima para a classificação do envolvimento em bullying, em termos de estar na posição de alvo, autor e alvo/autor. Assim, no presente estudo foram utilizados os seguintes critérios de classificação: (1) autor exclusivo: ter pontuação maior ou igual ao escore médio de autoria de violência e menor do que o escore médio para vitimização; (2) alvo exclusivo: ter pontuação maior ou igual à pontuação média para vitimização e menor do que o escore médio para autoria; (3) alvo-autor: ter pontuação acima ou igual ao escore médio em relação à vitimização e/ou autoria (Forlim et al., 2014). No presente estudo, para ser classificado como alvo de bullying, foi considerada a pontuação média de 14,6 pontos (máximo 76 pontos); já como autor de bullying, 13,5 pontos (máximo 52 pontos).

\section{Procedimento}

O estudo seguiu as normas e procedimentos éticos propostos pela Resolução 466/2012 do Conselho Nacional de Ética em Pesquisa envolvendo seres humanos. O projeto foi aprovado pelo Comitê de Ética em Pesquisa local (protocolo número 164.696), com autorização da direção das instituições envolvidas no estudo e prévia anuência da Secretaria de Educação do município. Todos os estudantes assinaram o Termo de Assentimento para participar do estudo e trouxeram os Termos de Consentimento Livre e Esclarecidos firmados pelos pais ou responsáveis legais.
A coleta de dados ocorreu no período de agosto a novembro de 2013, por meio da aplicação coletiva dos instrumentos em sala de aula. A administração foi realizada no final do período das atividades didáticas de rotina da escola, mediante agendamento prévio e autorização do professor responsável pela classe, de modo a não interferir nas ações programadas. O tempo despendido para o preenchimento dos instrumentos foi de, aproximadamente, 50 minutos. Após o autopreenchimento, todos os questionários foram colocados em envelopes lacrados sem identificação e depositados em uma urna, a fim de garantir o anonimato dos respondentes.

\section{Análise de Dados}

Após a coleta, os dados foram tabulados e armazenados em uma planilha eletrônica (Microsoft Excel). Os resultados foram submetidos à análise estatística por meio do programa Statistical Package for the Social Science (SPSS) para Windows versão 17.0. Na análise dos dados, foi utilizada estatística descritiva para cálculos de frequência, média e desvio-padrão.

O Teste Chi-quadrado e o Teste Exato de Fisher foram utilizados para avaliar as associações entre os diferentes tipos de envolvimento em bullying, as variáveis sociodemográficas (sexo, idade, raça, religião, com quem mora, relacionamento familiar, série, tipo e localização da escola e participação em festas) e o uso de drogas. Para a análise multivariada, todas as variáveis com valores de $p \leq 0,001$ foram consideradas. O modelo de regressão logística (método stepwise) avaliou a probabilidade do envolvimento em bullying como vítima (variável dependente) ocorrer em relação à idade, religião e com quem mora, como autor (variável dependente) ocorrer em função da idade e com quem mora e como alvo/autor (variável dependentes), em função da idade. Todos os testes estatísticos foram realizados com 5\% de significância, a partir de um Intervalo de Confiança (IC) de 95\%.

\section{RESULTADOS}

Os estudantes eram predominantemente do sexo feminino $(618-51,8 \%)$ apresentavam média de idade de 12,9 anos $(\mathrm{DP}=1,3)$, variando de 11 a 15 anos; cursavam o $6^{\circ}$ ano do Ensino Fundamental $(335-28,1 \%)$; eram de raça branca $(616-51,7 \%)$; residiam em casa própria $(820$ - 68,8\%); eram católicos (843 - 70,7\%); frequentavam festas/baladas uma a duas vezes por semana $(498-41,8 \%)$; moravam com pais/mães $(710-59,6 \%)$; mantinham um relacionamento familiar referido como muito bom (780 - 65,4\%); eram provenientes de escolas públicas (1.006 - 84,4\%); as quais são localizadas em zona urbana $(1.140-95,6 \%)$.

A Tabela 1 apresenta as diferenças entre as características sociodemográficas dos estudantes e o envolvimento em bullying. Variáveis como idade, religião, participação em festas e localização da escola se diferenciaram significativamente na amostra em relação ao tipo de envolvimento em bullying $(\mathrm{p} \leq 0,05)$. Entre os estudantes classificados como autores de bullying, observou-se predominância de: faixa etária entre 11 e 13 anos (174$55,2 \%)$, católicos $(224-71,1 \%)$ e que moravam em zona urbana (308 - 97,8\%). Ser vítima de bullying foi mais frequente em estudantes que frequentavam festas/baladas de uma a duas vezes por semana $(177-44,5 \%)$ e que moravam na zona urbana (389-97,7\%). Os adolescentes classificados como alvo/autor de bullying frequentavam festas/baladas de uma a duas vezes por semana $(67-54,5 \%)$ e moravam na zona urbana $(1.112-99,2 \%)$.

Quanto ao uso de drogas, $395(33,1 \%)$ experimentaram qualquer tipo de droga, $218(18,3 \%)$ haviam feito uso 
experimental de drogas (exceto álcool e tabaco), 284 (23,8\%)

fizeram uso de álcool e $58(5,0 \%)$ uso de tabaco no último mês.
Quase a metade dos adolescentes havia vivenciado alguma forma de bullying. Observou-se, também, que 70,1\% dos adolescentes vivenciaram situações de bullying, seja na

Tabela 1

Características sociodemográficas e classificação do bullying entre estudantes do ensino fundamental de um municipio do sul de Minas Gerais ( $N=1192)$

\begin{tabular}{|c|c|c|c|c|c|c|}
\hline \multirow{3}{*}{ Total } & \multicolumn{2}{|c|}{$\begin{array}{l}\text { Autor } \\
\text { (Sim) }\end{array}$} & \multicolumn{2}{|c|}{$\begin{array}{l}\text { Vítima } \\
\text { (Sim) }\end{array}$} & \multicolumn{2}{|c|}{$\begin{array}{l}\text { Alvo/Autor } \\
\quad \text { (Sim) }\end{array}$} \\
\hline & $\mathrm{N}$ & $\%$ & $\mathrm{~N}$ & $\%$ & $\mathrm{~N}$ & $\%$ \\
\hline & 315 & 26,4 & 398 & 33,4 & 123 & 10,3 \\
\hline \multicolumn{7}{|l|}{$\operatorname{Sexo}^{\mathrm{a}}$} \\
\hline Feminino & 159 & 50,5 & 206 & 51,8 & 60 & 48,8 \\
\hline Masculino & 156 & 49,5 & 192 & 48,2 & 63 & 51,2 \\
\hline \multicolumn{7}{|l|}{ Faixa etária ${ }^{a}$} \\
\hline $11-13$ anos & 174 & $55,2 *$ & 256 & 64,3 & 69 & 56,1 \\
\hline $14-15$ anos & 141 & 44,8 & 142 & 35,7 & 54 & 43,9 \\
\hline \multicolumn{7}{|l|}{$\operatorname{Raça}^{a}$} \\
\hline Branca & 164 & 52,1 & 202 & 50,8 & 64 & 52,0 \\
\hline Negra & 151 & 47,9 & 196 & 49,2 & 59 & 48,0 \\
\hline \multicolumn{7}{|l|}{ Religião } \\
\hline Católica & 224 & $71,1^{*}$ & 279 & 70,1 & 83 & 67,5 \\
\hline Espírita & 19 & 6,0 & 12 & 3,0 & 3 & 2,4 \\
\hline Ateu & 10 & 3,2 & 13 & 1,6 & 6 & 4,9 \\
\hline Evangélica & 62 & 19,7 & 181 & 22,8 & 31 & 25,2 \\
\hline \multicolumn{7}{|c|}{ Participação em festas/baladas } \\
\hline 1 a 2 vezes por semana & 131 & 41,6 & 177 & $44,5^{*}$ & 67 & $54,5^{*}$ \\
\hline 3 a 4 vezes por semana & 18 & 5,7 & 8,0 & 2,0 & 4 & 3,3 \\
\hline Uma vez por mês & 135 & 42,9 & 161 & 40,5 & 40 & 32,5 \\
\hline Outras & 31 & 9,8 & 52 & 13,1 & 12 & 9,8 \\
\hline \multicolumn{7}{|l|}{ Com quem mora } \\
\hline Pai e mãe & 179 & 56,8 & 234 & 58,8 & 71 & 57,7 \\
\hline Mãe & 82 & 26,0 & 101 & 25,4 & 31 & 25,2 \\
\hline Pai & 18 & 5,7 & 30 & 7,5 & 10 & 8,1 \\
\hline Amigos/Instituições & 2 & 0,6 & 3 & 0,8 & 1 & 0,8 \\
\hline Familiares & 34 & 10,8 & 30 & 7,5 & 10 & 8,1 \\
\hline \multicolumn{7}{|l|}{ Relacionamento familiar } \\
\hline Muito bom & 204 & 64,8 & 253 & 63,6 & 71 & 57,7 \\
\hline Bom & 78 & 24,8 & 108 & 27,1 & 39 & 31,7 \\
\hline Regular/Ruim/Muito ruim & 33 & 10,5 & 37 & 9,6 & 13 & 10,6 \\
\hline \multicolumn{7}{|l|}{ Série } \\
\hline $6^{\circ}$ ano & 84 & 26,7 & 122 & 30,7 & 36 & 29,3 \\
\hline $7^{\circ}$ ano & 71 & 22,5 & 113 & 28,4 & 29 & 23,6 \\
\hline $8^{\circ}$ ano & 73 & 23,2 & 72 & 18,1 & 26 & 21,1 \\
\hline $9^{\circ}$ ano & 87 & 27,5 & 91 & 22,9 & 32 & 26,0 \\
\hline \multicolumn{7}{|l|}{ Tipo de escola ${ }^{\mathrm{a}}$} \\
\hline Pública & 259 & 82,2 & 330 & 82,9 & 106 & 86,2 \\
\hline Particular & 56 & 17,8 & 68 & 17,1 & 17 & 13,8 \\
\hline \multicolumn{7}{|l|}{ Localização $^{\mathrm{a}}$} \\
\hline Urbana & 308 & $97,8^{*}$ & 389 & $97,7^{*}$ & 1112 & $99,2 *$ \\
\hline Rural & 7 & 2,2 & 9,0 & 2,3 & 1 & 0,8 \\
\hline
\end{tabular}

Nota: ${ }^{a}$ Teste Exato de Fisher. Teste de Chi-quadrado. $(* p \leq 0,05)$. 
condição de vítima $(33,4 \%)$, de autor $(26,4 \%)$ e, em menor proporção, alvo/autor (10,3\%).

A Tabela 2 apresenta as associações estatisticamente significantes entre bullying e uso de drogas no último mês $(\mathrm{p} \leq 0,05)$. A maioria dos adolescentes classificados como autor de bullying havia usado algum tipo de droga (exceto álcool e tabaco) $(727-82,9 \%)$, álcool $(89-28,3 \%)$ e cocaína $(12-3,8 \%)$. Apenas $248(31,2 \%)$ dos adolescentes classificados como vítimas de bullying haviam consumido algum tipo de droga. Por fim, entre os adolescentes caracterizados como autor/alvo em situação de bullying houve predomínio do uso de maconha $(10-8,1 \%)$, com diferenças estatisticamente significantes.

Quanto aos fatores associados, nota-se que ter idade de 12 anos $(\mathrm{OR}=1,4)$, ser ateu $(\mathrm{OR}=2,6)$ e morar em zona urbana $(\mathrm{OR}=2,3)$ aumentaram as razões de chances de ser vítima de bullying. Assim, considerando a situação de ser autor de bullying, as razões de chance aumentam quando se apresentam as seguintes características: ter idade de 15 anos $(\mathrm{OR}=1,7)$ e morar em zona urbana $(\mathrm{OR}=2,4)$. No caso de ser alvo/autor de bullying as chances dobram apenas em relação à idade - ter idade de 13 anos $(\mathrm{OR}=2,0)$.

Tabela 2

Distribuição dos estudantes do Ensino Fundamental segundo o uso de drogas (no último mês) e bullying entre estudantes do Ensino Fundamental de um municipio do sul de Minas Gerais $(N=1192)$

\begin{tabular}{|c|c|c|c|c|c|c|c|}
\hline & & \multicolumn{2}{|c|}{$\begin{array}{l}\text { Autor } \\
\text { (Sim) }\end{array}$} & \multicolumn{2}{|c|}{$\begin{array}{l}\text { Vítima } \\
\text { (Sim) }\end{array}$} & \multicolumn{2}{|c|}{$\begin{array}{l}\text { Alvo/Autor } \\
\text { (Sim) }\end{array}$} \\
\hline & & $\mathrm{N}$ & $\%$ & $\mathrm{~N}$ & $\%$ & $\mathrm{~N}$ & $\%$ \\
\hline \multirow{2}{*}{ Uso de qualquer droga } & Não & 598 & 68,2 & 546 & $68,8^{*}$ & 75 & 61,0 \\
\hline & Sim & 279 & 31,8 & 248 & 31,2 & 48 & 39,0 \\
\hline \multirow{2}{*}{$\begin{array}{l}\text { Uso de droga } \\
\text { (exceto álcool e tabaco) }\end{array}$} & Não & 727 & $82,9 *$ & 658 & 82,9 & 94 & 76,4 \\
\hline & Sim & 150 & 17,1 & 136 & 17,1 & 29 & 23,6 \\
\hline \multirow{2}{*}{ Álcool } & Não & 226 & 71,7 & 295 & 74,1 & 88 & 71,5 \\
\hline & Sim & 89 & $28,3^{*}$ & 103 & 25,9 & 35 & 28,5 \\
\hline \multirow{2}{*}{ Tabaco } & Não & 295 & 93,7 & 383 & 96,2 & 115 & 93,5 \\
\hline & Sim & 20 & 6,3 & 15 & 3,8 & 8 & 6,5 \\
\hline \multirow{2}{*}{ Maconha } & Não & 295 & 93,7 & 381 & 95,7 & 113 & 91,9 \\
\hline & Sim & 20 & 6,3 & 17 & 4,3 & 10 & $8,1^{*}$ \\
\hline \multirow{2}{*}{ Cocaína } & Não & 303 & 96,2 & 390 & 98,0 & 117 & 95,1 \\
\hline & Sim & 12 & 3,8 & 8 & 2,0 & 6 & 4,9 \\
\hline
\end{tabular}

Nota: Teste Exato de Fisher $\left({ }^{*} \mathrm{p} \leq 0,05\right)$.

\section{DISCUSSÃO}

O presente estudo buscou ampliar a compreensão acerca das eventuais relações entre bullying e uso de drogas em uma amostra robusta constituída por 1192 adolescentes escolares brasileiros. Na amostra investigada, quase a metade $(49,5 \%)$ dos adolescentes foram classificados como autor, vítima ou autor/vítima de bullying. Como esperado, foram obtidas altas porcentagens de adolescentes que se envolveram em situações de bullying, tanto na condição de vítima $(33,4 \%)$ como na de autor $(26,4 \%)$ e, em menor proporção, na posição de alvo/autor (10,3\%). Evidências mostram variações na prevalência de bullying que podem ocorrer de acordo com os diferentes instrumentos, metodologias e localidades utilizadas pelos estudos, o que pode explicar, em parte, as diferenças observadas nas prevalências encontradas nos vários estudos (Zsila et al., 2017). Independentemente de comparações com os resultados obtidos por outras pesquisas, os índices identificados no presente estudo são consideráveis e podem ser considerados preocupantes, uma vez que refletem uma característica social e cultural que parece ser típica da população local investigada. Esses achados são relevantes, tendo em vista que os efeitos negativos do bullying repercutem em diversos aspectos da vida do indivíduo, com efeitos potencialmente perturbadores sobre o desenvolvimento psicológico, adoção de comportamentos de saúde e aprendizagem acadêmica (Holt, Green, Tsay-Vogel, Davidson, \& Brown, 2017).

De fato, os achados deste estudo revelam prevalências bem superiores às observadas na literatura. Em uma pesquisa desenvolvida com estudantes de $6^{\circ}$ ao $9^{\circ}$ ano de escolas públicas em um município do interior paulista (São Carlos), 16\% dos estudantes declararam ter sido vítimas, $11 \%$ autores e $23 \%$ alvos/autores de bullying (Forlim et al., 2014). Em estudo realizado em Caxias do Sul, RS, com escolares de 11 a 14 anos, a prevalência encontrada foi de $10,2 \%$ na categoria vítima e $7,1 \%$ na de autor de bullying (Rech, Halpern, Tedesco, \& Santos, 2013). Já os resultados 
do levantamento realizado na cidade de Pelotas mostraram prevalência de envolvimento em situações de bullying da ordem de 17,6\% (Moura, Cruz, \& Quevedo, 2011).

As condições sociodemográficas e o contexto social (escola) são fatores importantes que interferem nas questões relacionadas ao desencadeamento e desfecho do bullying (Holt et al., 2017). Evidências apontam que situações que envolvem o bullying sempre existiram e que elas acontecem com maior freqüência em determinados ambientes escolares, em geral na Educação Infantil e no Ensino Fundamental, principalmente entre estudantes do $6^{\circ}$ ao $9^{\circ}$ ano (Forlim et al., 2014; Stelko-Pereira et al., 2010;).

A literatura destaca que, embora o bullying seja um problema social, acontece principalmente no ambiente escolar (Holt et al., 2017). Essa delimitação geográfica é um dos elementos cruciais para a definição do fenômeno (Stelko-Pereira et al., 2010). Na amostra investigada, os adolescentes apresentaram características peculiares em relação ao bullying, diferenciando-se em relação à idade, religião, participação em festas e localização da escola (Tabela 1).

Em relação à idade, em ambas as análises os adolescentes mais novos (12-13 anos) apresentaram maior probabilidade $(\mathrm{OR}=1,4)$ de serem vítimas ou alvo/autor de bullying. Entretanto, para os adolescentes em idade mais avançada (14 e 15 anos), as porcentagens foram maiores no grupo de autoria do bullying. Esses resultados corroboram os achados de estudos prévios, que mostram que há uma relação entre idade e bullying, com uma tendência de mudança de perfil e propensão a ser vítima ou autor à medida que aumenta a idade (Oliveira et al., 2016; Rech et al., 2013; Santos, Cabral-Chavier, Paiva, \& Leite-Cavalvante, 2014; Sebastião, 2009). O estudo revelou que, ao contrário das vítimas, os adolescentes autores eram mais velhos (13 a 15 anos) e estudavam em séries mais avançadas (Sebastião, 2009). Outras investigações também evidenciaram que adolescentes mais velhos apresentam maiores chances de serem agressores quando comparados aos mais novos (Oliveira et al., 2016; Rech et al., 2013).

Os achados do presente estudo também corroboram os resultados do levantamento nacional realizado com 60.973 estudantes de escolas públicas e privadas do território brasileiro. Esse levantamento mostrou que há uma relação inversa entre bullying e idade, ou seja, ser vítima de bullying é uma ocorrência mais comum em adolescentes mais novos (13 anos) e ser autor de bullying é mais frequente entre os mais velhos (acima de 15 anos) (Malta et al., 2010).

Na presente investigação, no modelo final de análise (multivariada), notou-se que adolescentes sem religião e residentes em zona urbana têm maiores chances de serem classificados como vítima, e adolescentes de escolas de zona urbana, como autor.

Religião, participação em festas e estudar na zona urbana foram variáveis associadas ao bullying. Além de aspectos psicossociais, as questões culturais dos adolescentes são aspectos determinantes para o envolvimento em situações de bullying. Quanto à vinculação religiosa, observa-se que a maioria dos estudantes católicos foi classificada como autores de bullying. A religião pode ser um modulador de certos comportamentos, notadamente os considerados desviantes ou negativos, pois oferece um sistema de valores que pauta as normas de conduta dos seguidores, favorecendo a mudança de atitude e o vislumbre de uma perspectiva de futuro, reduzindo, assim, a probabilidade de envolvimento em comportamentos de risco, delinquência e uso de substâncias psicoativas (Regnerus, 2003). Corroborando essa tendência, um estudo que avaliou 1.145 adolescentes de 11 a 15 anos da região sul do Brasil mostrou que a religião (vinculação religiosa e frequência de comparecimento aos rituais religiosos) foi um dos fatores de proteção para o desenvolvimento de transtornos de conduta, incluindo o bullying (Cruzeiro et al., 2008).

A participação do adolescente em festas/baladas (uma a duas vezes por semana) foi associada a ser vítima de bullying. Pode-se supor que a participação em festas, para os escolares de 12 a 15 anos que provavelmente são vítimas de bullying, pode denotar uma tentativa desses adolescentes de encontrarem espaços de sociabilidade e interação social por meio do convívio com os pares. $\mathrm{Na}$ etapa do desenvolvimento na qual o adolescente geralmente começa a frequentar festas e baladas, este pode estar mais vulnerável a sofrer a pressão dos pares e, assim, ficar exposto a julgamentos ou ser motivo de chacotas e intimidação. Se o adolescente já tem autoestima baixa, associada às transições psicossociais que são esperadas nessa fase do ciclo vital, pode se tornar alvo preferencial das provocações dos colegas, o que acaba por reforçar ainda mais a prática reiterada do bullying. A literatura a respeito desse tema ainda é escassa, o que sugere a necessidade de novos estudos que investiguem os processos psicológicos e comportamentais que desencadeiam e alimentam as situações de violência em contexto escolar, como o bullying.

Outro resultado que chamou a atenção nas três condições de envolvimento em bullying refere-se à localização da escola. Estudar em escolas situadas na zona urbana esteve associado significativamente ao bullying nas três condições (autor, vítima e alvo/autor). Esse achado sugere que o ambiente rural pode ser protetivo em relação ao bullying. Essa relação merece ser melhor explorada em investigações futuras. De fato, há um estudo que associa esse resultado às marcantes diferenças de condições, estilos e percepções de vida, que influenciariam o bem-estar geral e o monitoramento parental; assim, morar em zona rural parece funcionar como fator atenuante em relação ao envolvimento em bullying (Del-Duca, Garcia, Sousa, Oliveira, \& Nahas, 2010).

A associação entre bullying e localização da escola pode estar relacionada às condições de vida das famílias residentes na zona urbana e a maior exposição aos meios de comunicação de massa e aos apelos e imperativos da sociedade de consumo. Além disso, a precariedade do 
monitoramento dos pais, por estarem em grande parte do tempo envolvidos em atividades laborais, o modelo de disciplina privilegiado (estilo de imposição da autoridade parental) ou mesmo o estresse parental pela sobrecarga de trabalho tendem a afetar negativamente o desenvolvimento dos filhos adolescentes, que podem se sentir desamparados e compelidos a adotar como estratégias de relacionamento entre os pares a agressão ou o isolamento, mediadas por sentimentos de raiva, competitividade e repúdio às diferenças (Oliveira, Silva, Yoshisaga, \& Silva, 2015).

As características sociais e culturais associadas ao bullying culminam por modular o comportamento dos adolescentes e o modo como estes respondem às diferentes demandas culturais relacionadas à alteridade e diversidade, fomentando o desrespeito às diferenças que emergem nos relacionamentos com os pares. Assim, atentar a essas vulnerabilidades e oferecer o apoio necessário para coibilas pode favorecer a neutralização desse tipo de violência escolar que tem vulnerabilizado os adolescentes (Oliveira et al., 2016).

Foram encontradas associações positivas entre uso de drogas e bullying (Tabela 2). Os adolescentes autores de bullying usaram algum tipo de droga (exceto álcool e tabaco) $(82,9 \%)$, álcool $(28,3 \%)$ ou cocaína $(3,8 \%)$. Por outro lado, $31,2 \%$ dos adolescentes que foram vítimas de bullying haviam consumido algum tipo de droga. Entre os adolescentes classificados como autor/alvo de bullying houve predomínio do uso de maconha $(8,1 \%)$. Independentemente do tipo de droga utilizada, a associação com o bullying (sofrido ou praticado) se fez presente. No entanto, esperavase encontrar, na análise multivariada, potenciais razões de chance entre quem consumiu algum tipo de substância e o bullying, tanto em termos de autoria como de vitimização, uma vez que a literatura evidencia essa associação (Durand et al., 2013; Houbre et al., 2006; Zsila et al., 2017), o que não se confirmou na presente investigação.

Um estudo de metanálise apontou associação entre bullying e uso de drogas entre adolescentes escolares, de modo que o fato de ser autor aumentou em três vezes as chances de usar drogas, enquanto ter sido vítima de bullying dobrou as chances para o consumo (Valdebenito et al., 2015) em níveis mais severos que o outro (Radlliff et al., 2012). Além disso, a sobreposição de comportamentos agressivos e o uso de substâncias foram relacionadas a tentativas fracassadas dos adolescentes para enfrentarem situações de vitimização e rejeição por pares (Carlyle \& Steinman, 2007).

Pesquisas sugerem que algumas vítimas podem não ter habilidades de enfrentamento adequadas, o que pode ser ainda agravado pelo fato de não receberem suporte para gerenciar a experiência de vitimização (Goebert, Else, Matsu, Chung-Do, \& Chang, 2011). Adolescentes vitimizados geralmente apresentam pensamentos intrusivos, que foram positivamente associados ao tabagismo, o que sugere que eles podem utilizar a nicotina como forma de redução da ansiedade gerada pelas situações de bullying a que são expostos, ou para incrementar sua imagem social entre os pares, de modo a elevar sua autoestima (Houbre et al., 2006).

$\mathrm{Na}$ amostra investigada, ser alvo/autor de bullying foi associado significativamente ao uso de maconha. $\mathrm{Na}$ literatura, são poucos os estudos que avaliam de forma discriminada o comportamento de quem sofre e de quem pratica o bullying. Entretanto, levantamentos realizados na Finlândia e nos EUA revelam que ter sido alvo/autor aumenta três vezes as chances de usar bebidas alcoólicas, sete vezes as chances de usar drogas, além de favorecer a tendência ao uso de tabaco (Kaltiala-Heino et al., 2000; Radliff et al., 2012).

Todavia, até onde se pôde observar na literatura revisada, não foi encontrado qualquer estudo de levantamento que mostrasse a relação entre o uso de drogas e o envolvimento com bullying na população de adolescentes do Ensino Fundamental. Essa é uma das fortalezas do presente estudo, pois os adolescentes que relataram uso de drogas (exceto álcool e tabaco), álcool e cocaína foram associados à autoria. No entanto, o uso de qualquer tipo de droga foi maior entre adolescentes que foram vítimas, e o uso de maconha mostrou ser mais significativo entre os escolares que haviam sido alvo/autor de bullying.

Apesar de ter sido identificada, no presente estudo, uma baixa porcentagem de adolescentes alvos/autores de bullying, um estudo sugere que eles apresentam risco elevado para o desenvolvimento de problemas de comportamento quando comparados a vítimas e autores exclusivos, pois carregam um efeito cumulativo dos resultados negativos desse fenômeno de uma dupla dimensão de vítima e perpetrador (Durand et al., 2013).

O bullying, na sua tríplice dimensão (alvo, autor e alvo/autor), pode variar de acordo com a idade, religião, local de residência, participação em festas e uso de drogas. As associações apontadas no presente estudo fornecem subsídios para que profissionais das áreas de saúde e educação, bem como pais e comunidade, possam se articular para promover uma rede de apoio e proteção ao adolescente vulnerável. Em suma, os resultados obtidos confirmam que é de extrema importância que o contexto escolar se preocupe em identificar e avaliar sistematicamente o bullying, visto que esse fenômeno envolve problemas comportamentais complexos e multifatoriais que podem gerar impacto na saúde física, psicológica e socioeducacional dos estudantes (Alckmin-Carvalho, Izbicki, Fernandes, \& Melo, 2014). A produção de conhecimento nessa área é de crucial importância para fundamentar estratégias de prevenção e promoção de saúde no contexto escolar, uma vez que estudos que relacionaram o bullying e o uso de drogas ainda se mostram incipientes no Brasil, como já discutido.

Algumas limitações do presente estudo devem ser consideradas. Uma delas é que, por esta ser uma pesquisa de delineamento transversal, não é possível determinar a direcionalidade entre as variáveis bullying e o uso de drogas. 
Assim, são necessárias investigações futuras, com diferentes desenhos metodológicos (inclusive prospectivos, com recorte longitudinal), para melhor compreender a relação existente entre os fenômenos, tanto na esfera educacional como na de saúde pública, a fim de fornecer evidências para que esses setores fortaleçam a promoção de saúde, antes mesmo do início da adolescência.

Como fortalezas do estudo, destacam-se o uso de um delineamento metodológico robusto, a mensuração da relação entre uso de drogas e o envolvimento em bullying por meio da utilização de instrumentos padronizados (DUSI R e EVE-E) para aferição das variáveis do estudo, a utilização de uma amostra representativa de adolescentes estudantes do Ensino Fundamental de um município brasileiro, inclusive com a inclusão de estudantes oriundos de escolas localizadas na zona rural. Em relação às potenciais implicações dos achados para a prática clínica, os resultados fornecem subsídios para o cuidado integral em saúde envolvendo uma abordagem multiprofissional, contribuindo, desse modo, para o desenvolvimento de estratégias preventivas que fomentem uma cultura social de enfrentamento das situações de bullying em associação com o uso de drogas entre adolescentes escolares, de modo a minimizar riscos à saúde desse segmento da população.

\section{CONSIDERAÇÕES FINAIS}

O objetivo principal do presente estudo foi avaliar as relações entre uso de drogas e envolvimento em bullying entre adolescentes no contexto escolar. Os achados obtidos permitem concluir que o bullying foi associado tanto a características sociodemográficas quanto ao uso de drogas. Consumir algum tipo de droga (exceto álcool e tabaco), álcool e cocaína foi associado à autoria de bullying. Ser vítima de bullying foi associado ao uso de droga (exceto álcool e tabaco), enquanto ser alvo/autor foi relacionado ao uso de maconha. Esses dados chamam a atenção, principalmente pela descoberta de que quase a metade dos adolescentes escolares vivenciaram alguma forma de bullying. Além disso, a idade (12 anos), religião (ateu) e estudar em escola de zona urbana aumentam as razões de chance para vitimização. Adolescentes com idade mais avançada (15 anos) e aqueles que estudam em escola de zona urbana têm chances elevadas de serem alvos/autores, assim como ter 13 anos aumenta as razões de chance de ser autor.

Apesar de não se poder estabelecer uma relação de direcionalidade do nexo causal entre as variáveis investigadas, os dados fornecem elementos que podem subsidiar programas de promoção e prevenção de saúde no contexto escolar. Considera-se que tanto o envolvimento em bullying na condição de autor, vítima ou alvo/autor como o uso de substâncias psicoativas são fenômenos comuns e com magnitude de ocorrência relevante em adolescentes escolares, o que permite inferir os impactos consideráveis que desencadeiam na vida desses jovens. Assim, é preciso repensar as políticas públicas de educação, saúde e cuidado direcionadas a essa população, provendo intervenção precoce e assistência adequada para atenuar os potenciais agravos decorrentes da exposição.

\section{REFERÊNCIAS}

Alckmin-Carvalho, F., Izbicki, S., Fernandes, L. F. B., \& Melo, M. H. D. S. (2014). Estratégias e instrumentos para a identificação de bullying em estudos nacionais. Avaliação Psicológica, 13(3), 343-350.

Andrade, S. S. C. D. A., Yokota, R. T. D. C., Bandeira de Sá, N. N., Silva, M. M. A. D., Araújo, W. N. D., Mascarenhas, M. D. M., \& Malta, D. C. (2012). Relação entre violência física, consumo de álcool e outras drogas e bullying entre adolescentes escolares brasileiros. Cadernos de Saúde Pública, 28(9), 1725-1736. doi:10.1590/S0102-311X2012000900011

Bradshaw, C. P., Waasdorp, T. E., Goldweber, A., \& Johnson, S. L. (2013). Bullies, gangs, drugs, and school: Understanding the overlap and the role of ethnicity and urbanicity. Journal of Youth and Adolescence, 42(2), 220-234. doi:10.1007/s10964012-9863-7

Carlyle, K. E., \& Steinman, K. J. (2007). Demographic differences in the prevalence, co-occurrence, and correlates of adolescent bullying at school. Journal of School Health, 77(9), 623-629. doi:10.1111/j.1746-1561.2007.00242.x

Carlini, E. L. A., Noto, A. R., Sanchez, Z. van der M., Carlini, C. M. A., Locatelli, D. P., Abeid, L. R. ... Moura, Y. G. (2010). VI Levantamento nacional sobre o consumo de drogas psicotrópicas entre estudantes do Ensino Fundamental e
Médio das redes pública e privada de ensino nas 27 capitais brasileiras. São Paulo: CEBRID/UNIFESP e SENAD. Retirado de https:/www.cebrid.com.br/wp-content/uploads/2012/10/ VI-Levantamento-Nacional-sobre-o-Consumo-de-DrogasPsicotr\%C3\%B3picas-entre-Estudantes-do-EnsinoFundamental-e-M\%C3\%A9dio-das-Redes-P\%C3\%BAblicae-Privada-de-Ensino-nas-27-Capitais-Brasileiras.pdf

Cook, C. R., Williams, K. R., Guerra, N. G., Kim, T. E., \& Sadek, S. (2010). Predictors of bullying and victimization in childhood and adolescence: A meta-analytic investigation. School Psychology Quarterly, 25(2), 65-83. doi:10.1037/a0020149

Cruzeiro, A. L. S., Silva, R. A. D., Horta, B. L., Souza, L. D. D. M., Faria, A. D., Pinheiro, R. T., ... \& Ferreira, C. D. (2008). Prevalência e fatores associados ao transtorno da conduta entre adolescentes: Um estudo de base populacional. Cadernos de Saúde Pública, 24(9), 2013-2020.

Del Duca, G. F., Garcia, L. M. T., Sousa, T. D., Oliveira, E. D., \& Nahas, M. V. (2010). Insatisfação com o peso corporal e fatores associados em adolescentes. Revista Paulista de Pediatria, 28(4), 340-346.

de Micheli, D., \& Formigoni, M. L. O. (2000). Screening of drug use in a teenage Brazilian sample using the Drug Use Screening Inventory (DUSI). Addictive Behaviors, 25(5), 683-691. doi:10.1016/S0306-4603(00)00065-4 
de Micheli, D., \& Formigoni, M. L. O. (2002). Psychometric properties of the Brazilian version of the Drug Use Screening Inventory. Alcoholism: Clinical and Experimental Research, 26(10), 1523-1528. doi:10.1111/j.1530-0277.2002.tb02451.x

Durand, V., Hennessey, J., Wells, D. S., Crothers, L. M., Kolbert, J. B., Lipinski, J., \& Hughes, T. L. (2013). Bullying and substance use in children and adolescents. Journal of Addiction Research and Therapy, 4(4), 1-6. doi:10.4172/2155-6105.100015

Farrington, D., \& Baldry, A. (2010). Individual risk factors for school bullying. Journal of Aggression, Conflict and Peace Research, 2(1), 4-16. doi:10.5042/jacpr.2010.0001

Forlim, B. G., Stelko-Pereira, A. C., \& Williams, L. C. D. A. (2014). Relação entre bullying e sintomas depressivos em estudantes do ensino fundamental. Estudos de Psicologia (Campinas), 31(3), 367-375. doi:10.1590/0103-166X2014000300005

Goebert, D., Else, I., Matsu, C., Chung-Do, J., \& Chang, J. Y. (2011). The impact of cyberbullying on substance use and mental health in a multiethnic sample. Maternal and Child Health Journal, 15(8), 1282-1286. doi:10.1007/s10995-010-0672-x

Holt, M. K., Green, J. G., Tsay-Vogel, M., Davidson, J., \& Brown, C. (2017). Multidisciplinary approaches to research on bullying in adolescence. Adolescent Research Review, 2(1), 1-10. doi:10.1007/s40894-016-0041-0

Horta, C. L., Horta, R. L., Mester, A., Lindern, D., Weber, J. L. A., Levandowski, D. C., \& Lisboa, C. S. D. M. (2018). Bullying e uso de substâncias psicoativas na adolescência: Uma revisão sistemática. Ciência \& Saúde Coletiva, 23(1), 123-140. doi:10.1590/1413-81232018231.20932015

Houbre, B., Tarquinio, C., Thuillier, I., \& Hergott, E. (2006). Bullying among students and its consequences on health. European Journal of Psychology of Education, 21(2), 183-208. doi:10.1007/BF03173576

Instituto Brasileiro de Geografia e Estatística. (2016). Pesquisa Nacional de Saúde do Escolar - PeNSE. Rio de Janeiro, RJ: IBGE.

Kaltiala-Heino, R., Rimpelä, M., Rantanen, P., \& Rimpelä, A. (2000). Bullying at school: An indicator of adolescents at risk for mental disorders. Journal of Adolescence, 23(6), 661-674. doi:10.1006/jado.2000.0351

Kim, M. J., Catalano, R. F., Haggerty, K. P., \& Abbott, R. D. (2011). Bullying at elementary school and problem behaviour in young adulthood: A study of bullying, violence and substance use from age 11 to age 21. Criminal Behaviour and Mental Health, 21(2), 136-144. doi:10.1002/cbm.804

Lazarus, R. S. (1993). Coping theory and research: Past, present, and future. Psychosomatic Medicine, 55(3), 234-247.

Luk, J. W., Wang, J., \& Simons-Morton, B. G. (2012). The cooccurrence of substance use and bullying behaviors among US adolescents: Understanding demographic characteristics and social influences. Journal of Adolescence, 35(5), 1351-1360. doi:10.1016/j.adolescence.2012.05.003

Luukkonen, A. H. (2010). Bullying behaviour in relation to psychiatric disorders, suicidality and criminal offences: A study of under-age adolescent inpatients in Northern Finland. Oulun yliopisto (Doctoral Dissertation). University of Oulu, Finland.

Malta, D. C., Silva, M. A. I., Mello, F. C. M. D., Monteiro, R. A., Sardinha, L. M. V., Crespo, C., ... Porto, D. L. (2010). Bullying in Brazilian schools: Results from the National School-based Health Survey (PeNSE), 2009. Ciência \& Saúde Coletiva, 15(2), 3065-3076. doi:10.1590/S1413-81232010000800011

Moura, D. R., Cruz, A. C. N., \& Quevedo, L. A. (2011). Prevalência e características de escolares vítimas de bullying. Jornal de Pediatria, 87(1), 19-23. doi:10.2223/JPED.2042

Nansel, T. R., Craig, W., Overpeck, M. D., Saluja, G., \& Ruan, W. J. (2004). Cross-national consistency in the relationship between bullying behaviors and psychosocial adjustment.
Archives of Pediatrics \& Adolescent Medicine, 158(8), 730736. doi:10.1001/archpedi.158.8.730

Nesello, F., Sant'Anna, F. L., Santos, H. G. D., Andrade, S. M. D., Mesas, A. E., \& González, A. D. (2014). Características da violência escolar no Brasil: Revisão sistemática de estudos quantitativos. Revista Brasileira de Saúde Materno Infantil, 14(2), 119-136. doi:10.1590/S1519-38292014000200002

Niemelä, S., Brunstein-Klomek, A., Sillanmäki, L., Helenius, H., Piha, J., Kumpulainen, K., ... Sourander, A. (2011). Childhood bullying behaviors at age eight and substance use at age 18 among males: A nationwide prospective study. Addictive Behaviors, 36(3), 256-260. doi:10.1016/j.addbeh.2010.10.012

Oliveira, W. A., Silva, J. L. D., Yoshinaga, A. C. M., \& Silva, M. A. I. (2015). Interfaces entre família e bullying escolar: Uma revisão sistemática. Psico USF, 20(1), 121-132. doi:10.1590/141382712015200111

Oliveira, W. A., Silva, M. A. I., Silva, J. L., Mello, F. C. M., do Prado, R. R., \& Malta, D. C. (2016). Associations between the practice of bullying and individual and contextual variables from the aggressors' perspective. Jornal de Pediatria, 92(1), 32-39. doi:10.1016/j.jpedp.2015.06.002

Oliveira, W. A., Silva, J. L., Querino, R. A., Santos, C. B., Ferriani, M. G. B. C., Santos, M. A., \& Silva, M. A. I. (2018a). Revisão sistemática sobre bullying e família: Uma análise a partir dos sistemas bioecológicos. Revista de Salud Publica, 20(3), 396403. doi:10.15446/rsap.v20n3.47748

Oliveira, W. A., Silva, J. L., Santos, M. A., Hayashida, M., Caravita, S. C. S., \& Silva, M. A. I. (2018b). Interações familiares de estudantes em situações de bullying. Jornal Brasileiro de Psiquiatria, 67(3), 151-158. doi:10.1590/00472085000000204

Olweus, D. (1993). Bullying at school: What we know and what we can do. Maiden, MA: Blackwell.

Olweus, D. (2013). School bullying: development and some important challenges. Annual Review of Clinical Psychology, 9, 751-780. doi:10.1146/annurev-clinpsy-050212-185516

Pranjić, N., \& Bajraktarević, A. (2010). Depression and suicide ideation among secondary school adolescents involved in school bullying. Primary Health Care Research \& Development, 11(04), 349-362. doi:10.1017/S1463423610000307

Radliff, K. M., Wheaton, J. E., Robinson, K., \& Morris, J. (2012). Illuminating the relationship between bullying and substance use among middle and high school youth. Addictive Behaviors, 37(4), 569-572. doi:10.1016/j.addbeh.2012.01.001

Rech, R. R., Halpern, R., Tedesco, A., \& Santos, D. F. (2013). Prevalência e características de vítimas e agressores de bullying. Journal de Pediatria, 89(2), 164-170. doi:10.1016/j. jped.2013.03.006

Regnerus, M. D. (2003). Moral communities and adolescent delinquency. The Sociological Quarterly, 44(4), 523-554. doi:10.1111/j.1533-8525.2003.tb00524.x

Santos, J. A. D., Cabral-Xavier, A. F., Paiva, S. M., \& LeiteCavalcanti, A. (2014). Prevalência e tipos de bullying em escolares brasileiros de 13 a 17 anos. Revista de Salud Pública, 16(2), 173-183. doi:10.15446/rsap.v16n2.30302

Schnohr, C., \& Niclasen, B. W. L. (2006). Bullying among Greenlandic schoolchildren: Development since 1994 and relations to health and health behaviour. International Journal of Circumpolar Health, 65(4), 305-312.

Sebastião, J. (2009). Violência na escola: Uma questão sociológica. Revista Interacções, 13, 35-62.

Silva, J. L., Oliveira, W. A., Bono, E. L., Dib, M. A., Bazon, M. R., \& Silva, M. A. I. (2016). Associações entre o bullying escolar e conduta infracional: Revisões sistemáticas de estudos longitudinais. Psicologia: Teoria e Pesquisa, 32(1), 81-90. doi:10.1590/0102-37722016012241081090 
Stelko-Pereira, A. C., Williams, L. C. A., \& Freitas, L. C. (2010). Validade e consistência interna do Questionário de Investigação de Prevalência de Violência Escolar: Versão estudantes. Avaliação Psicológica, 9(3), 403-411.

Tharp-Taylor, S., Haviland, A., \& D'Amico, E. J. (2009). Victimization from mental and physical bullying and substance use in early adolescence. Addictive Behaviors, 34(6), 561-567. doi:10.1016/j.addbeh.2009.03.012

Ttofi, M. M., Farrington, D. P., Lösel, F., \& Loeber, R. (2011). The predictive efficiency of school bullying versus later offending: A systematic/meta-analytic review of longitudinal studies. Criminal Behaviour and Mental Health, 21(2), 80-89. doi:10.1002/cbm.808

Ttofi, M. M., \& Farrington, D. P. (2008). Bullying: Short-term and long-term effects, and the importance of defiance theory in explanation and prevention. Victims and Offenders, 3(2-3), 289-312. doi:10.1080/15564880802143397

Valdebenito, S., Ttofi, M., \& Eisner, M. (2015). Prevalence rates of drug use among school bullies and victims: A systematic review and meta-analysis of cross-sectional studies. Aggression and Violent Behavior, 23, 137-146. doi:10.1016/j.avb.2015.05.004 Zsila, Á., Orosz, G., Király, O., Urbán, R., Ujhelyi, A., Jármi, É., ... Demetrovics, Z. (2017). Psychoactive substance use 